\title{
L'emploi et la protection sociale dans l'agriculture au filtre des rapports de genre
}

Les salariées agricoles aux $\mathrm{XIX}^{\mathrm{e}}$ et $\mathrm{XX}^{\mathrm{e}}$ siècles

Employment and Social Protection in Agriculture Through the Lens of Gender

Relations. Agricultural Female Workers in the 19th and 20th Centuries

Jean-Louis Escudier

\section{(2) OpenEdition}

Journals

\section{Édition électronique}

URL : http://journals.openedition.org/travailemploi/7392

DOI : 10.4000/travailemploi.7392

ISSN : 1775-416X

Éditeur

DARES - Ministère du Travail

Édition imprimée

Date de publication : 1 janvier 2017

Pagination : 73-97

ISSN : 0224-4365

Référence électronique

Jean-Louis Escudier, «L'emploi et la protection sociale dans l'agriculture au filtre des rapports de genre », Travail et Emploi [En ligne], 149 | janvier-mars 2017, mis en ligne le 11 juillet 2019, consulté le 09 septembre 2019. URL : http://journals.openedition.org/travailemploi/7392 ; DOI : 10.4000/ travailemploi.7392 


\title{
L'emploi et la protection sociale dans l'agriculture au filtre des rapports de genre
}

\author{
Les salariées agricoles aux XIX ${ }^{\mathrm{e}}$ et $\mathrm{XX}^{\mathrm{e}}$ siècles
}

\author{
Jean-Louis Escudier"
}

\begin{abstract}
Cet article examine les rapports entre les conditions d'emploi et de rémunération des salariées agricoles et les modalités de leur accès à la protection sociale en s'appuyant sur l'exploitation de sources administratives (enquêtes agricoles, rapports sur les opérations des sociétés de secours mutuels, statistique annuelle des institutions d'assistance, enquêtes de l'Institut national de la statistique et des études économiques [Insee]) et le dépouillement de livres de comptes d'exploitations agricoles. Des années 1850 à 1918, la protection sociale des femmes salariées agricoles se résume à de rares dispositifs d'assistance car les structures mutualistes renâclent à les intégrer. À partir des années 1930, les salariées agricoles sont affiliées aux assurances sociales mais les taux de cotisation les pénalisent. La mise en place des allocations familiales agricoles institue alors un sursalaire dont le montant n'est pas encore de nature à les exclure du système productif. Après la Libération, le salaire des ouvrières agricoles est revalorisé mais leur emploi devient de plus en plus occasionnel en raison de la mécanisation des principaux travaux. La revalorisation des allocations familiales et l'attribution de l'allocation de salaire unique (complément de prestations familiales alloué aux ménages percevant un seul salaire) encouragent le retrait quasi total des femmes du salariat agricole permanent.
\end{abstract}

$\mathrm{L}$ e salariat féminin a donné lieu à nombre de recherches centrées sur l'emploi (OMnès, 1997 ; Letablier, Nicole-Drancourt, 2007 ; MARuani, Meron, 2012) ou sur les écarts de rémunération entre hommes et femmes (LEMIÈRE, 2006 ; MEURS, Ponthieux, 2000 ; Muller, 2012 ; Silvera, 2014). Toutefois, les approches historiques se sont jusqu'à présent principalement focalisées sur des professions spécifiques

\footnotetext{
* Laboratoire montpelliérain d'économie théorique et appliquée (Lameta), université de Montpellier ; escudier@ lameta.univ-montp1.fr.
} 
telles que les salariées de l'industrie (GuILBERT, 1966 ; Downs, 2001 ; Escudier, 2015) ou les domestiques (MArTin-Fugier, 2004). Parallèlement, la question de la protection sociale différenciée selon le genre a fait l'objet d'investigations de la part d'historien.ne.s et de sociologues (BATTAGLIOla, 1999, 2000 ; Cova, 1997 ; THÉBAUD, 1986 ; CHAINEAUD, 2012), qui privilégient l'approche par les risques sociaux (maternité, maladie, vieillesse). En revanche, l'examen croisé, sur le long terme, de la rémunération des femmes et de leur accès à la protection sociale reste un espace à explorer. Dans la mesure où la protection sociale « à la française » se caractérise au $\mathrm{XX}^{\mathrm{e}}$ siècle par une généralisation de l'assurance professionnelle obligatoire ${ }^{1}$, nous proposons d'en apprécier les implications différenciées pour les femmes et les hommes salariés.

Bien qu'elles aient séculairement constitué une catégorie professionnelle numériquement importante, ni les salariées agricoles ni leurs conditions de travail et de rémunération n'ont été spécifiquement étudiées. Tout au plus apparaissent-elles fugacement dans des publications s'intéressant aux agricultrices (BECOUARN, 1975 ; BERLAN, PAINVIN, 1979 ; BARTHEZ, 1982 ; BESSIÈRE, 2006) ou aux hommes salariés agricoles (FARCY, HuBSCHER, 1996 ; PIÉGAY, 2007 ; DARPEIX, 2010). Le salariat agricole féminin est une entité difficile à cerner dans la mesure où il ne répond qu'imparfaitement aux critères retenus pour identifier la population active, à savoir un emploi permanent dans une monoactivité. Or cette population féminine s'inscrit largement dans la pluriactivité, la transversalité des statuts et, toujours, aux franges de la sphère professionnelle et de la sphère domestique. La catégorie la plus durablement prise en considération dans les sources statistiques est celle des « servantes de ferme », salariées logées et nourries, intégrées à la communauté de vie autant qu'à l'exploitation agricole proprement dite. Bien d'autres femmes travaillent moyennant salaire dans les exploitations agricoles. Rémunérées au temps, qualifiées de " journalières », plus rarement d' " ouvrières agricoles » mais dans la plupart des cas enregistrées dans les livres de comptes sous le seul vocable de «femmes », ces travailleuses sont employées pour des durées très variables selon les lieux, les saisons et les besoins de chaque exploitation. Les salariées agricoles n'étant pas une catégorie statistique stabilisée sur la période, nous précisons le périmètre de notre analyse au fil du texte, dans chacune des trois parties qui le composent.

\section{0-1918 : des salariées assignées à l'assistance sociale}

En dépit de leur nombre, de leur disponibilité temporelle et de l'intensité de leur travail, les salariées agricoles restent jusqu' au sortir de la Première Guerre mondiale mal identifiées. Alors même qu'elles sont très médiocrement rémunérées, employées

1. L'assurance professionnelle obligatoire repose sur le principe d'une cotisation fixe ou proportionnelle au salaire acquittée pour partie par l'employeur et pour partie par le salarié. 
souvent par intermittence et tenues à l'écart du mouvement mutualiste, leur protection sociale, jusqu'alors inexistante, relève, à partir des années 1890, du champ de l'assistance ${ }^{2}$.

\section{La salariée agricole, parent pauvre de la Statistique}

Le corpus des quatre enquêtes agricoles réalisées dans la seconde moitié du XIX siècle $(1852,1862,1882$ et 1892) est un matériau statistique aussi précieux que délicat à exploiter. En l'absence de tout fichier émanant d'organismes sociaux, de déclarations de récolte, de déclarations d'emploi, de contrats collectifs de travail, toutes les statistiques relatives à l'emploi (durée, salaires en espèces, prestations en nature, etc.) relèvent d'informations éparses, de la parole de notables que les agents en charge de la centralisation des données de l'enquête se contentent de généraliser. De plus, la partition entre emploi permanent, emploi temporaire et emploi saisonnier n'est pas encore normée. Par-delà leur variabilité, les catégories statistiques réfèrent toujours à un emploi centré sur une seule activité, exercé à temps plein et rémunéré au temps, modèle qui recoupe parfaitement l'emploi de l'homme adulte, l'emploi féminin étant quant à lui davantage sujet à l'intermittence, à la pluriactivité et à la rémunération à la tâche. Par une confrontation entre les sources primaires (relevés communaux) et les comptes consolidés à l'échelle du canton, du département et du pays, nous avons mis en évidence la fragilité de ces statistiques pour la main-d'œuvre en général et féminine en particulier (EsCUDIER, 2014, pp. 60-73). D'une enquête à l'autre, le nombre de salariées agricoles dénombrées varie de 400000 à 900000 sans autre explication. Pour les exploitants agricoles, intéressés au premier chef par le coût de la main-d'œuvre salariée, l'évaluation du travail nécessaire pour cultiver un hectare importe plus que le dénombrement précis et le genre des salarié.e.s. Dès lors, à l'image d'un Pierre LÉVÊQUE (1996), historien de la Bourgogne contemporaine exploitant les données de l'enquête de 1852, bien des auteurs négligeront l'examen de cette catégorie de travailleuses si difficile à cerner.

$\mathrm{La}$ « volatilité » de l'emploi des journalières agricoles contraste avec l'emploi masculin. Une journalière « permanente » est alors employée au cours d'une année entre 150 et 220 jours contre 300 jours pour un journalier agricole. Quel que soit le type de production animale ou végétale, les femmes apparaissent comme une main-d'œuvre potentiellement disponible, toujours mobilisée pour les temps forts (plantation, traitements, fenaisons, moissons, vendanges, etc.) et sollicitée « en pointillé » le restant de l'année. La norme salariale des journalières agricoles, est, jusqu'en 1946, le « demisalaire » de l'homme sans qualification ${ }^{3}$. L'intermittence de l'emploi agricole féminin

2. La mutualité sociale relève du champ de l'assurance librement consentie ; on parle généralement d'assurance mutuelle. Relèvent de l'assistance toutes les prestations sociales non contributives, c'est-à-dire sans contrepartie financière de la part des bénéficiaires.

3. Pour la seconde moitié du XIX ${ }^{\mathrm{e}}$ siècle, cette norme salariale transparaît de manière récurrente dans les livres de comptes des exploitations agricoles ; elle est explicitement mentionnée dans les contrats collectifs conclus à la suite des 
est considérée comme « normale », voire « naturelle » et son oubli dans les statistiques officielles l'est tout autant. Les réponses formulées par les professeurs départementaux d'agriculture dans le cadre de l'enquête nationale de 1910 sur les salaires agricoles sont édifiantes à cet égard. À la question «Quelle est la durée approximative du chômage dans une année pour les salariés agricoles fixes de chacune des régions de votre département ? » (MinistÈrE DE L'AGRICULTURE, 1912, p. 35), à une exception près, ces fonctionnaires répondent en se référant exclusivement à la main-d'œuvre masculine.

\section{La Mutualité, une protection sociale masculine et urbaine}

Le décret du 26 mars 1852, acte fondateur de la Mutualité dite "impériale », traduit une conception éminemment masculine de la protection sociale. Les femmes peuvent être admises et percevoir une indemnité proportionnelle à leur cotisation en cas d'accident du travail ou de maladie. En revanche, en aucun cas, elles ne peuvent participer à l'administration ni aux délibérations des sociétés de secours mutuels (SSM ${ }^{4}$. La tonalité étant clairement affichée, la majorité des SSM seront des « sociétés d'hommes seuls » : en 1892 , les sociétés mixtes représentent $27,3 \%$ des sociétés mutuelles approuvées, les sociétés masculines 69,8\% et les sociétés féminines seulement 2,9\% (DREYFus, 2001, p. 114). Ces dernières furent créées à l'initiative d'enseignantes et d'ouvrières du textile ou de la chimie.

En 1886, la cotisation annuelle moyenne s'élève dans les sociétés approuvées à 11,45 francs pour les femmes et 15,09 francs pour les hommes. Le ministre de l'Intérieur juge cette cotisation féminine " manifestement insuffisante ${ }^{5}$ » au prétexte qu'elle ne couvre pas l'intégralité des dépenses obligatoires des femmes faisant appel aux services mutualistes. C'est là ignorer les disparités salariales puisque rapporté à leur salaire, l'effort financier consenti par les femmes pour leur couverture sociale est incontestablement plus prononcé : leurs 11,45 francs de cotisations représentent de dix à douze journées de travail des salariées agricoles, soit de 5 à $8 \%$ de leurs revenus annuels, alors que trois à quatre journées de travail suffisent aux hommes pour acquitter 15,09 francs de cotisation.

Avec 1222000 membres adultes cotisants au 31 décembre 1898 sur une population active totale estimée à 19 millions d'individus, la Mutualité ne parvient pas à imposer son modèle de protection sociale. De plus, entre 1871 et 1900, la part des femmes parmi les mutualistes progresse modestement : de $14,8 \%$ à $18,7 \%$ des

grèves viticoles des années 1904-1912. Jusqu'en 1939, les syndicats affiliés à la Fédération nationale des travailleurs de l'agriculture de la Confédération générale du travail (CGT) intégreront le demi-salaire féminin dans leurs négociations avec les représentants des employeurs.

4. Constituées sur une base géographique ou professionnelle, les sociétés de secours mutuels regroupent des adhérents qui, moyennant une cotisation mensuelle, bénéficient de prestations sociales afférentes aux risques « santé », « accident du travail », « invalidité », et, pour une minorité d'entre elles, au risque « vieillesse ».

5. Ministère de l'Intérieur (1889), Rapport sur les opérations des sociétés de secours mutuels pendant l'année 1886 présenté à M. le Président de la République par M. Constans, ministre de l'Intérieur, Melun, Imprimerie administrative, p. 63. 
adhérent.e.s ${ }^{6}$ et, parmi elles, les agricultrices, salariées ou non, sont très marginales car l'implantation de la Mutualité est nettement moindre en milieu rural, comme le déplorent régulièrement les responsables du mouvement. Or la protection des risques sociaux ne s'adresse qu'aux seuls adhérents, la notion d'ayants droit étant, jusqu'aux années 1930, étrangère au mouvement mutualiste. Les femmes sont de fait largement exclues de ce type de couverture sociale. Les statistiques relatives à la Côte-d'Or sont éloquentes : au 31 décembre 1857, ce département comptait 60 SSM fédérant 4512 membres actifs, dont seulement 149 femmes ; en 1905, les 120 SSM de ce département grouperont 7800 hommes et seulement 380 femmes et enfants (MARTIN, MARTENOT, 1909, p. 476). Les femmes ne constituent que 3,3\% des membres actifs en 1857 et 4,6\% en 1905.

Avec la nouvelle loi-cadre de la Mutualité, celle du $1^{\mathrm{er}}$ avril 1898, dite de la Mutualité républicaine, les femmes ne sont plus légalement exclues de toute implication dans la gestion mutualiste ; elles peuvent faire partie des SSM et en créer, les femmes mariées étant exonérées de l'autorisation de leur mari. Ce nouveau cadre législatif moins misogyne incite certaines SSM à accorder une prestation forfaitaire pour accouchement et des sociétés d'hommes seuls à se transformer en sociétés mixtes (TouCAS-TRUyen, 2004). L'ouverture des SSM aux femmes reste toutefois modeste. Dans l'Hérault, où le mouvement mutualiste est précocement et solidement implanté, les 51402 mutualistes se répartissent en 1913 en 37908 hommes, 10183 femmes et 3311 enfants. Au mieux, le taux de couverture sociale des femmes adultes atteint $10 \%$, et est nettement inférieur en milieu rural. De fait, la plupart des responsables mutualistes locaux usèrent longtemps d'un argument spécieux pour écarter les frais inhérents à la maternité du champ de la protection mutualiste : la maternité n'étant pas une maladie, elle ne saurait relever d'une prise en charge médicale. Autre objection, apparemment plus sérieuse : les femmes étant réputées plus souvent malades que les hommes, leur intégration mettrait en péril l'équilibre financier des mutuelles. Or, si le nombre annuel de jours de maladie des femmes est supérieur à celui des hommes, les indemnités journalières qu'elles perçoivent sont nettement inférieures car calculées sur la base de leur rémunération annuelle. Il n'est qu'un domaine (les frais pharmaceutiques) dans lequel les femmes mutualistes soient plus dispendieuses que les hommes ${ }^{7}$.

La part des femmes adhérentes se stabilise autour de $20 \%$ dans la première décennie du Xx ${ }^{\mathrm{e}}$ siècle. Au 31 décembre 1927, à la veille de la mise en place des assurances sociales, le nombre de femmes mutualistes atteint pour la première fois le cap du million ; leur nombre ayant augmenté plus rapidement que celui des hommes, elles constituent alors $23,6 \%$ des mutualistes ${ }^{8}$. Cette timide ouverture des SSM en direction des femmes ne modifie pas la donne. Les problèmes de fond, à savoir la

\footnotetext{
6. Ministère de l'Intérieur et des Cultes (1903), Rapport sur les opérations des sociétés de secours mutuels pendant l'année 1900 présenté à M. le Président de la République par M. Combes, président du Conseil, ministre de l'Intérieur et des Cultes, Melun, Imprimerie administrative, pp. 14-15.

7. Ibid., pp. 50-51.

8. Cité par Bulletin des sociétés de secours mutuels, nº 1-2, janvier-février 1930, pp. 130-131.
} 
faiblesse des moyens financiers de la Mutualité et son incapacité à se rapprocher des couches sociales les plus modestes, ne sont pas résolus. La plupart des SSM n'intégreront des femmes qu'à partir de 1930, lorsque la mise en place des assurances sociales obligatoires les contraindra à assurer les salarié.e.s sans distinction de sexe.

\section{Aux femmes, l'assistance, aux hommes, les prémices de la protection sociale assise sur le salaire}

L'article $1^{\text {er }}$ de la loi sur l'Assistance médicale gratuite (AMG) du 15 juillet 1893 énonce : « Les femmes en couches sont assimilées à des malades ${ }^{9}$. » Par cette formule pour le moins maladroite, sinon désobligeante, les femmes font une entrée massive et durable dans le champ de l'assistance. L'AMG rencontre immédiatement une large audience puisque le nombre d'indigents inscrits atteint 2178000 individus en 1912, dont une majorité de femmes. L'AMG offre aux plus démunies, notamment aux mères célibataires, séparées ou divorcées, l'accès aux soins ambulatoires et hospitaliers. Mais en milieu rural comme en milieu urbain, bon nombre de femmes inscrites sur les listes de l'AMG sont des épouses de mutualistes. Le caractère genré de cette législation a jusqu'ici été largement ignoré par l'historiographie de la protection sociale. Aucune contribution de l'ouvrage Différence des sexes et protection sociale (XIX ${ }^{e}-X X^{e}$ siècles) (Auslander, ZANCARINI-Fournel, 1995), ne traite par exemple de cette loi. Pour un spécialiste de la recherche en histoire médicale aussi averti qu'Olivier FAURE (1984, p. 604), « la légère surreprésentation des femmes [dans l'AMG] est normale et compense le moindre accès des femmes à la mutualité et à l'hôpital ». Or cette inscription des femmes dans l'assistance n'est pas un épiphénomène mais une constante historique. À tous les âges, les femmes sont surreprésentées dans l'octroi de l'AMG : en 1912 , elles représentent $60,7 \%$ des bénéficiaires ${ }^{10}$.

En l'absence de congé de maternité rémunéré et, le plus souvent, de toute contribution masculine aux tâches domestiques et fonctions parentales, la maternité peut engendrer la précarité. Le risque est manifeste dans les familles dépourvues de salaire masculin pour cause de décès, d'invalidité ou de séparation du couple. Or, en 1896, la proportion de femmes adultes seules (célibataires ou veuves) varie selon les départements de 30 à $40 \%$ (BouRDELAIS, 1981). La précarité guette également la plupart des ménages de journaliers en cas de perte du salaire féminin souvent indispensable à l'équilibre budgétaire familial. Dès lors, grossesse, accouchement et allaitement sont loin d'entraîner systématiquement l'interruption du travail agricole et des activités domestiques. Les enfants sont sevrés très précocement car la femme doit participer aux travaux des champs. À la fin du XIX ${ }^{e}$ siècle, l'entreprise champenoise Moët \& Chandon offre à la naissance de chaque enfant le berceau et la layette (DAVID, 1897, p. 101). Nonobstant cette contribution, les ouvrières travaillent jusqu' au jour de l'accouchement

9. Journal officiel, 18 juillet 1893, p. 3681.

10. Ministère du Travail, de l'Hygiène, de l'Assistance et de la Prévoyance sociales, Statistique générale de la France (1914), Statistique annuelle des institutions d'assistance, année 1912, Paris, Imprimerie nationale, p. X. 
et reprennent souvent leur emploi moins de huit jours plus tard. De l'aveu même du directeur général, cette reprise d'activité précipitée est cause de pathologies (CHANDON DE BRIAILLES, 1897, p. 257). Feignant d'ignorer que ni son entreprise ni une institution sociale ne compensent le manque à gagner de ces salariées, il leur reproche de ne pas respecter le repos postnatal de dix jours. La crainte de la maternité susceptible de remettre en cause un équilibre financier toujours fragile aurait généré en certains lieux, sinon des formes de malthusianisme, à tout le moins des modes de régulation des naissances afin d'éviter l'accouchement au moment des grands travaux agricoles (fenaisons, moissons, vendanges) (MARTIN, MARTENOT, 1909, p. 543).

La loi du 27 novembre 1909 garantissant le maintien de l'emploi des femmes en couches durant huit semaines consécutives pendant la période précédant et suivant l'accouchement s'applique aux salariées de l'agriculture comme à celles de l'industrie, disposition qui n'allait pas de soi dans un contexte où la législation sociale agricole était constamment dérogatoire. La loi sur le repos des femmes en couches promulguée le 17 juin 1913 constitue le second volet des mesures de protection maternelle et infantile. Elle interdit l'emploi des femmes au cours des quatre semaines suivant leur accouchement dans les établissements industriels et commerciaux. Si le repos n'est pas obligatoire pour les salariées agricoles et les employées de maison, toute salariée de nationalité française se voit accorder une allocation journalière pendant la période de repos précédant et suivant immédiatement ses couches. L'appréciation d'Anne Cova, qualifiant de «faibles » les montants de cette allocation journalière « car, en 1913, le salaire d'une ouvrière est supérieur à 2 francs par jour » (CovA, 1997, p. 163), vaut pour les seules ouvrières de l'industrie jouissant d'un emploi stable et continu. Pour les ouvrières agricoles intermittentes, dont le salaire journalier n'excédait pas 1,25 à 1,50 franc, un tel soutien financier est appréciable. Assurément, cette allocation journalière, complétée par la suite par les primes départementales à la natalité (loi du 29 juin 1918 et décret du 30 avril 1920), contribue à retarder le retour à l'emploi après accouchement. Par contre, le travail jusqu'à la veille de l'accouchement reste de mise. Les récits relatant des naissances dans les champs ou, tout au moins, dès le retour précipité au logis ne sont pas rares. L'enfant une fois mis au monde, l'absence de structure de prise en charge socialisée se fait cruellement sentir. Excepté dans les familles élargies où elle peut se faire aider, l'accouchée suspend les tâches ménagères durant un laps de temps réduit à deux ou trois jours. Pour concilier allaitement et emploi agricole, bien des femmes retournent aux champs avec leur nourrisson. Dès le déclenchement de la Première Guerre mondiale, une allocation journalière de 1,25 franc majorée de 50 centimes par enfant de moins de 16 ans est octroyée aux épouses de mobilisés. Si cette aide est notoirement insuffisante pour les ménages urbains confrontés aux prix élevés des produits de première nécessité, son montant est supérieur au revenu mensuel moyen des journalières agricoles.

Seule forme assurancielle émergeant avant la Première Guerre mondiale, les retraites ouvrières et paysannes (ROP) instaurées par la loi du 5 avril 1910 visent à accorder aux salarié.e.s de plus de 65 ans une pension proportionnelle à leurs années 
de cotisation. Les cotisations annuelles obligatoires sont fixées à 9 francs pour les hommes, 6 francs pour les femmes et 4,50 francs pour les mineurs de moins de 18 ans ; l'employeur est astreint à un versement annuel équivalent. Bien que relativement modestes, ces taux de contribution sociale pénalisent doublement les journalières agricoles : d'une part, ils ne correspondent pas à la réalité de la norme genrée du demisalaire horaire telle qu'elle s'impose en agriculture dans les années 1850 ; d'autre part, ils ignorent le caractère intermittent de l'emploi de ces salariées. Au total, ces dernières doivent acquitter une cotisation sociale égale aux deux tiers de celle versée par leurs homologues masculins alors que leur rémunération annuelle n'excède pas le quart ou le tiers de celle des hommes ouvriers agricoles non qualifiés. Par deux arrêts en date des 11 décembre 1911 et 22 juin 1912, la Cour de cassation supprime l'obligation du précompte, c'est-à-dire du prélèvement à la source par l'employeur de la cotisation salariale. Dès lors, les ROP deviennent de facto facultatives et une minorité de salariés et d'agriculteurs y adhèrent. L'intermittence et le statut de revenu d'appoint de l'emploi féminin expliquent qu'une proportion encore moindre de travailleuses y souscrit.

\section{8-1945 : de l'assistance au sursalaire familial}

Au cours de cette période, le salariat agricole féminin est toujours aussi difficile à cerner. La grande enquête agricole dite de 1929 (en fait, l'essentiel des informations fut collecté en 1932 et 1933) recense 312815 salariées permanentes et 348319 salariées temporaires (Ministère DE L'AgRiculture, 1937, pp. 632-633). À l'évidence, comme nous allons le voir, ces chiffres sont sous-évalués. La partition entre main-d'œuvre familiale et main-d'œuvre salariée d'une part, entre salariat et non-salariat d'autre part, reste incertaine ; la partition entre emploi permanent et emploi temporaire n'est pas davantage explicitée.

\section{Une rémunération toujours faible des journalières et servantes de ferme}

L'application de la loi du 15 décembre 1922 étendant aux exploitations agricoles la législation sur les accidents de travail permet d'obtenir une statistique départementale des salaires journaliers et annuels par catégories de travailleurs agricoles. Ces données sont collectées par chaque préfet après consultations des responsables socioprofessionnels. Elles sont publiées annuellement au Journal officiel et sont ensuite reprises par les services de la Statistique générale de la France (SGF) dans le cadre de son enquête annuelle sur l'évolution des salaires. Ces données nous paraissent fragiles à plusieurs titres. La moyenne nationale résulte de situations locales extrêmement disparates : d'un département à l'autre, le salaire des journalières comme celui des servantes de ferme varie dans une proportion de un à deux. Par ailleurs, le rapprochement des salaires journalier et annuel laisse à penser que l'emploi annuel des journalières est largement surévalué : la moyenne sur les années 1924 à 1932 s'établit à 256 jours pour 
les journalières contre 275 pour les journaliers (ANONYME, 1933, pp. 238-240). Nos investigations d'ordre monographique ${ }^{11}$ débouchent sur des taux d'occupation annuel bien moindres. Au vu de ces études, il semblerait que la journalière "permanente » soit employée entre 150 et 200 jours par an durant les années 1920 et sensiblement moins au cours de la décennie suivante. Son salaire suit toujours la norme du demisalaire masculin. En dépit de ces réserves, cette statistique montre que, dans tous les départements, le revenu annuel de la servante de ferme excède celui de la journalière.

Les années d'entre-deux-guerres marquent l'avènement des assurances sociales et des allocations familiales obligatoires. Dès lors, les salariées agricoles ne sont plus seulement assignées à des dispositifs d'assistance même si l'AMG occupe encore un rôle non négligeable. En 1925, parmi les adultes de 16 à 59 ans, les femmes représentent 56,6\% des bénéficiaires de l'AMG. Les conditions d'attribution de l'allocation d'assistance aux femmes en couches sont assouplies : en 1925, les 294168 bénéficiaires constituent plus de la moitié des parturientes de cette année-là et parmi elles une majorité sont des salariées agricoles ${ }^{12}$. Relèvent également d'un dispositif d'assistance sociale les quelque 650000 veuves de guerre percevant une pension versée par l'État au titre de la loi du 31 mars 1919.

\section{L'affiliation des salariées agricoles aux assurances sociales}

Avec les lois du 5 avril 1928 et du 30 avril 1930, la protection sociale change de nature en France. Les formes d'assistance (assistance médicale gratuite, aide directe des bureaux de bienfaisance, assistance aux vieillards, infirmes et incurables, etc.) et la libre adhésion aux sociétés de secours mutuels trouvent leurs limites. L'assurance professionnelle obligatoire assise sur le salaire permet une bien meilleure couverture des risques sociaux (ESCUDIER, 1999). Mais dans quelle mesure les femmes salariées agricoles bénéficient-elles de cette protection sociale d'un nouveau type ?

En vertu de la loi du 5 avril 1928, deviennent obligatoirement assurés sociaux tou.te.s les salarié.e.s de moins de 60 ans dont la rémunération en espèces et en nature est inférieure à 15000 francs s'ils n'ont pas d'enfants, à 18000 et 20000 francs s'ils en ont un ou deux. Les ressources des assurances sociales sont constituées par un versement de $10 \%$ de la masse salariale : $5 \%$ à la charge de l'assuré, retenus sur sa paye, et $5 \%$ à la charge de l'employeur auquel incombe le versement de cette double contribution. Par cette affiliation au régime des assurances sociales, les salarié.e.s agricoles obtiennent des droits à l'assurance maladie et à la retraite. L'assurance maladie couvre les frais de traitement et d'hospitalisation pour l'assuré, son conjoint et leurs enfants non salariés âgés de moins de 16 ans. Expression symbolique du statut d'ayants droit dans le cadre des assurances sociales obtenu par des millions de femmes, l'allocation d'allaitement se traduit par l'octroi de 100 francs les deux

11. Essentiellement à partir de livres de comptes de grandes exploitations viticoles.

12. Ministère du Travail, de l'Hygiène, de l'Assistance et de la Prévoyance sociales, Statistique générale de la France (1928), Statistique annuelle des institutions d'assistance, année 1925, Paris, Imprimerie nationale, p. 90. 
premiers mois de la vie de l'enfant, 75 francs le troisième, 50 francs du quatrième au sixième mois, 25 francs du septième au neuvième mois et enfin 15 francs du dixième au douzième mois. La représentation socioprofessionnelle des exploitants agricoles s'oppose à cette législation sociale. Le 21 juin 1929, la toute jeune assemblée des présidents de chambres d'agriculture vote à l'unanimité un vœu tendant à ne maintenir l'obligation de protection assurantielle que pour le risque vieillesse, excluant donc les risques «maladie » et «invalidité » (LAGRAVE, LECLERC, 1996, p. 279). Les réseaux politiques sont activés pour faire reculer le gouvernement ou, faute d'y parvenir, pour retarder l'application du dispositif et en alléger la charge pour les employeurs. Un fort mouvement de révolte animé par le sulfureux Henri Dorgères ${ }^{13}$ engage les employeurs à refuser toute cotisation obligatoire (PAXTON, 1996, pp. 272-276). Le décret du 30 octobre 1935 déterminant le régime des assurances sociales applicables aux travailleurs agricoles a des effets contrastés pour les deux sous-catégories de salariées agricoles. Alors que la rémunération des journalières agricoles continue à se situer au niveau de la moitié du salaire masculin, il leur est imposé une cotisation forfaitaire correspondant à $80 \%$ de celle des salariés agricoles. En revanche, les servantes de ferme, dont le salaire est établi sur une base annuelle, ne sont pas lésées dans la mesure où elles travaillent plus que les 240 jours par an pris comme référence pour l'établissement de cette cotisation annuelle (tableau 1).

TABLEAU 1 - Régime des assurances sociales applicables aux salarié.e.s de l'agriculture en 1935

En francs

\begin{tabular}{lcccccc}
\hline Catégories & \multicolumn{3}{c}{ Cotisations journalières } & \multicolumn{3}{c}{ Cotisations annuelles } \\
\hline & Assuré & Employeur & Total & Assuré & Employeur & Total \\
\hline Enfants de moins de 16 ans & & & & & & \\
et ouvriers à capacité réduite & 0,30 & 0,30 & 0,60 & 72 & 72 & 144 \\
Femmes & 0,40 & 0,40 & 0,80 & 96 & 96 & 192 \\
Hommes & 0,50 & 0,50 & 1,00 & 120 & 120 & 240 \\
\hline
\end{tabular}

Source : Journal officiel du 31 octobre 1935, p. 11607.

Une recherche récente a mis l'accent sur les avancées sociales en direction des veuves durant les années d'entre-deux-guerres (Chaineaud, 2012). Mais pour les salariées agricoles, ces avancées furent plus virtuelles que réelles. Ainsi, l'attribution d'une pension de retraite est subordonnée à une durée de cotisation. Les assuré.e.s âgé.e.s de 60 ans dont le compte individuel aura été crédité pendant 30 ans au moins de cotisations annuelles atteignant 100 francs pour les hommes et 60 francs pour les femmes, ont droit à une pension de retraite égale à vingt fois la cotisation moyenne

13. De son vrai nom Henri-Auguste d'Halluin, Henri Dorgères (1897-1985) fonde en 1929 un Comité de défense paysanne contre les assurances sociales instaurées par la loi du 5 avril 1928. En 1934, proche des thèses de l'extrême droite, il organise plusieurs mouvements d'action paysanne, dont les Comités de défense paysanne qui eurent une influence essentiellement dans le Nord et l'Ouest de la France. 
versée au titre de l'assurance vieillesse ${ }^{14}$. Ce seul énoncé situe l'étendue des difficultés rencontrées par l'immense majorité des femmes rurales pour accéder à cette pension.

L'orientation essentiellement masculine de la Mutualité tend à s'estomper devant le caractère familial de la protection sociale qu'institue l'assurance professionnelle obligatoire : le nombre de femmes et d'enfants bénéficiant des prestations mutualistes progresse rapidement. Un certain nombre de SSM modifient même leur intitulé pour afficher cette prise en charge de l'ensemble de la cellule familiale (PRACA, 2000). Dans un contexte de contraction du taux de salarisation des femmes ${ }^{15}$, cette transformation majeure pèse sur les rapports de genre. La grande majorité des femmes bénéficient d'une couverture sociale au titre d'ayants droit et non d'assurés sociaux. En effet, l'application des assurances sociales contre lesquelles les milieux agrariens s'étaient élevés incite bon nombre d'employeurs agricoles à ne pas déclarer une partie des salariées, qu'elles soient domestiques, journalières ou saisonnières. Ces pratiques illégales prennent d'autant plus d'ampleur qu'elles obtiennent l'assentiment des salarié.e.s.

\section{Les allocations familiales agricoles comme sursalaire familial}

La question du sursalaire familial, évoquée dès les premières années du $\mathrm{Xx}^{\mathrm{e}}$ siècle par les courants natalistes, est réactivée au sortir de la Première Guerre mondiale. Au sein du monde agricole, la viticulture fait office d'élément moteur en raison de l'importance qu'y occupe le salariat et de la mobilisation plus forte de ses salarié.e.s. Les allocations familiales agricoles trouvent leur origine en 1919 lorsque le syndicat des grands crus classés du Médoc constitue une caisse commune destinée à verser un surcroît de rémunération aux salariés agricoles ayant charge de famille. À lui seul, l'intitulé de cette caisse en exprime la philosophie : «Caisse agricole de sursalaire familial de la région bordelaise ». Au sein de chaque terroir, chaque caisse adopte ses modalités de financement (en fonction des superficies cultivées, du nombre de salariés occupés) et édicte ses conditions d'octroi des prestations (GoldMAN, 1982). En Gironde, les employeurs acquittent une cotisation mensuelle de 3 francs par salarié homme, femme ou enfant de plus de 13 ans. En contrepartie, les ménages de salariés perçoivent 10 francs par mois pour un enfant de moins de 13 ans, 20 francs pour deux enfants et 35 francs pour trois. Mais cette contribution paraît élevée aux exploitants n'employant pas de familles nombreuses. Jugeant plus avantageux de pourvoir individuellement au payement d'allocations familiales afin d'en décider seuls du montant et des bénéficiaires potentiels, les gestionnaires des domaines les plus prestigieux, tels Château Latour et Château Lafite, refusent alors d'adhérer à la caisse commune. Fondée en 1924 et financée par une cotisation de 5,95 francs par hectare cultivé, la Caisse de compensation agricole de l'Hérault verse une prime à la naissance accordée dès le premier enfant et une allocation pour famille nombreuse d'un montant mensuel

14. Article 7 du décret du 30 octobre 1935.

15. Le taux d'activité des femmes en France régresse de 42,3 \% en 1921 à 34,2 \% en 1936 (MARUANI, MERON, 2012, p. 40). 
de 10 francs pour le troisième enfant, de 25 francs à partir du quatrième, de 45 francs à partir du cinquième ${ }^{16}$. Mais cette institution sociale ne rencontre aucun écho hors du cercle étroit des plus grands propriétaires viticoles (EsCUDIER, 2016, p. 212). Dans les Pyrénées-Orientales, les prestations octroyées par la caisse constituée le 20 février 1927 par trois syndicats professionnels agricoles sont plus attractives ${ }^{17}$. L'allocation mensuelle est cumulable : 15 francs pour le deuxième enfant, 20 francs pour le troisième, 30 francs pour le quatrième, 40 francs pour le cinquième et au-delà. La prime de maternité s'élève à 100 francs lors de la naissance du deuxième enfant, 150 francs pour le troisième, 200 francs pour le quatrième et les suivants. Peu enclin à la cogestion sociale, le syndicat des ouvriers agricoles reste dans l'expectative et les propriétaires ne sont pas davantage enthousiastes. En dépit de leurs limites manifestes, ces institutions ont le mérite d'exister alors que la majeure partie du territoire national en reste dépourvu. En 1925, le ministère de l'Agriculture institue une Commission des allocations familiales en agriculture « chargée d'étudier toutes les questions relatives à la diffusion des caisses d'allocations familiales intéressant l'agriculture ${ }^{18} »$. Malgré cela, en 1930, seulement 34 départements sont pourvus de caisses d'allocations familiales agricoles regroupant en tout 3011 exploitants cotisants versant 1169491 francs (Ministère de L'Agriculture, 1937, pp. 730-731).

La loi du 11 mars 1932 rendant obligatoires les allocations familiales à la charge des employeurs entre en vigueur dès l'année suivante dans les secteurs du commerce et de l'industrie. En revanche, le législateur renvoie à des décrets ultérieurs le soin de déterminer les modalités et les délais de mise en application de la loi dans l'agriculture, décrets que les gouvernements successifs ne s'empressent pas d'édicter. Le décret portant règlement d'administration publique pour l'application aux exploitations agricoles de la loi du 11 mars 1932 est enfin promulgué le 5 août 1936. Les exploitants sont sommés de s'organiser pour verser des allocations familiales dans les plus brefs délais. Dès lors, tous les départements métropolitains intègrent le régime obligatoire des allocations familiales, à partir du 16 novembre 1936 pour les premiers d'entre eux, au $1^{\text {er }}$ juillet 1937 pour les derniers.

Si les exploitants obtempèrent, leur représentation syndicale négocie avec succès : le taux des allocations familiales agricoles sera inférieur à celui des prestations accordées aux ménages relevant des secteurs de l'industrie et du commerce. Le taux de ces allocations, établi en fonction des prestations allouées par les caisses de compensations préexistantes, est extrêmement variable d'un département à l'autre. Maximales dans l'Aube (où elles atteignent 85 francs pour un ménage ayant deux enfants à charge et 200 francs pour trois enfants à charge), elles ne sont que de 30 francs par mois en Haute-Loire pour deux enfants à charge et de 45 francs en Corse pour trois

16. Archives départementales de l'Hérault, 7 M 1005, tract produit par la Caisse de compensation agricole de l'Hérault, 1925 .

17. Archives départementales des Pyrénées-Orientales, 10 M 76, Statuts et règlement intérieur de la Caisse de Compensation pour Allocations Familiales des agriculteurs roussillonnais, 1927.

18. Arrêté du 28 août 1925, Journal officiel, 11 septembre 1925. 
enfants à charge ${ }^{19}$. Quoi qu'il en soit, les modalités d'octroi de ces prestations (non subordonnées à des conditions de ressources et/ou à un retrait du marché du travail) et leur montant (l'équivalent monétaire de deux à quatre journées de travail par mois pour deux enfants à charge et de trois à six journées pour trois enfants à charge) ne sont pas de nature à inciter les femmes à se retirer du marché de l'emploi.

Dès le printemps 1937, les dirigeants de la Fédération nationale des travailleurs de l'agriculture (FNTA) de la CGT réunifiée tiennent force meetings pour réclamer que le taux des allocations familiales des ouvriers agricoles soit relevé au niveau de celui des salariés de l'industrie ${ }^{20}$. Une telle mobilisation traduit le désir de la grande majorité des ouvriers agricoles syndiqués de tendre vers le modèle de l'homme gagnepain (male breadwinners). Dans le cadre de l'encouragement national aux familles nombreuses, le décret du 19 juin 1939 accorde un supplément d'allocations familiales aux ménages de salariés agricoles de nationalité française ayant au moins trois enfants : 120 francs pour le troisième enfant, 360 francs pour le quatrième et 540 francs pour le cinquième et les suivants. Le décret relatif à la famille et à la natalité françaises en date du 29 juillet 1939 revêt une importance majeure. Par ses 167 articles, il a vocation à conférer pour quelques décennies à la politique familiale un cadre de référence durable, qui survive aux changements politiques et institutionnels éventuels, ce qui lui valut le titre de «Code de la famille ${ }^{21} »$. Le décret liste une série d'aides financières (primes à la première naissance, réévaluation des allocations familiales) et son chapitre III est intitulé « Dispositions spéciales à la famille paysanne ${ }^{22}$ ». Enfin, la loi du 29 mars 1941 institue une allocation de salaire unique au bénéfice de familles dont les enfants ont la nationalité française, allocation dont le montant est nettement plus substantiel que celui de l'allocation de mère au foyer accordée depuis avril $1939: 20 \%$ du salaire moyen départemental pour un enfant de moins de 5 ans, $25 \%$ de ce même salaire pour deux enfants à charge, $30 \%$ pour trois enfants ou plus.

\section{5-1980 : la salariée agricole éclipsée par l'homme gagne-pain}

L'intensification de la politique nataliste dès la Libération se traduit par de fortes incitations financières. Revalorisées, les prestations familiales deviennent attractives pour les couches populaires. En milieu rural bien plus qu'en milieu urbain, une forte proportion de femmes se replie définitivement sur la sphère domestique dès le lendemain de leur mariage.

19. Arrêté du ministère du Travail du 14 novembre 1936, Journal officiel, 20 novembre 1936, pp. 12049-12050.

20. Archives départementales de l'Hérault, 7 M 1006, Vœu exprimé par 67 syndicats d'ouvriers agricoles affiliés à la CGT, document dactylographié, s.d. [1937].

21. Journal officiel, 30 juillet 1939, pp. 9607-9626.

22. Articles 50 à 74 du décret du 29 juillet 1939. 


\section{Un revenu salarial annuel toujours réduit}

En vertu de l'ordonnance du 7 juillet 1945 relative aux commissions paritaires de travail en agriculture, le salaire de l'ouvrière agricole équivaut désormais à $80 \%$ du salaire masculin de base. Mais l'adoption d'un salaire minimum change à nouveau la donne. En énonçant que le salaire minimum interprofessionnel garanti (Smig) s'applique « aux travailleurs de l'un et l'autre $\operatorname{sexe}^{23} »$, le décret du 9 octobre 1950 consacre une conception novatrice des rapports économiques de genre. Le secteur agricole se voit par ailleurs appliquer des taux de salaire inférieurs, selon les zones, de 20 à $32 \%$ du Smig fixé pour l'industrie et le commerce. Au grand dam d'une fraction des représentants socioprofessionnels, ce salaire minimum spécifique (couramment qualifié de Smag, salaire minimum agricole garanti) contraint les employeurs agricoles à majorer la rémunération horaire de leur personnel féminin permanent et temporaire.

Le rattrapage salarial induit par le Smag doit être nuancé. Certes, entre 1951 et 1957, le salaire brut des servantes de ferme comme celui des domestiques hommes logés et nourris progresse davantage que celui des journaliers agricoles (MARCHE, 1958, p. 41), mais il s'agit là de catégories de travailleurs jusqu'alors particulièrement défavorisées. En ce qui concerne les journalières agricoles, les protocoles salariaux comprennent un seul tarif «femmes » alors que le salaire masculin fait l'objet d'une grille de rémunération intégrant des qualifications professionnelles, ouvriers volants, contremaîtres, tractoristes et cavistes étant nettement mieux rémunérés que le simple journalier. De plus, les écarts de durée d'emploi pèsent fortement sur les revenus respectifs de l'ouvrier et de l'ouvrière agricole. D'ailleurs, les services de l'Insee distinguent désormais clairement emploi permanent ( 200 heures ou plus par mois) et emploi temporaire, qui est lui-même subdivisé en deux sous-catégories : l'emploi saisonnier (binage des betteraves, vendanges, cueillette des fruits, etc.) et l'emploi occasionnel, qui «n'offre aucun caractère spécifique et peut-être exercé à toute période de l'année » (PujoL, 1969, p. 62). En 1968, les femmes sont quatre fois plus nombreuses à occuper un emploi agricole permanent à temps partiel qu'à temps complet. Le phénomène perdure : en 1980, alors que les femmes représentent 13,9\% des salarié.e.s agricoles permanent.e.s, elles totalisent $35 \%$ des unités de travail agricole ${ }^{24}$ (BIEGANSKI et al., 1981, p. 39).

Conséquence directe du fait qu'elles travaillent surtout à temps partiel, en 1964, la rémunération mensuelle des ouvriers agricoles non qualifiés ni logés ni nourris excède de 75,9 \% celle des ouvrières non qualifiées alors que leur salaire horaire est « seulement » supérieur de 32,2 \%. Si les écarts se restreignent quelque peu en 1968, ils restent significatifs : les ouvriers non qualifiés ni logés ni nourris gagnent chaque

23. Décret $n^{\circ}$ 50-1264 du 9 octobre 1950, Journal officiel, 10 octobre 1950.

24. Les unités de travail agricole ou unités de travail humain résultent de l'agrégation des temps annuels consacrés à l'exploitation, qu'il s'agisse de temps complet ou de temps partiel, de travailleurs permanents ou de travailleurs saisonniers. Il s'agit donc d'une notion proche de celle d'équivalent temps plein. 
mois 49,2 \% de plus que les ouvrières non qualifiées et « seulement » 26,7\% quand on considère le salaire horaire (tableau 2).

\section{TABLEAU 2 - Salaire brut moyen des salarié.e.s agricoles ni logé.e.s ni nourri.e.s occupant un emploi permanent $(1964,1966$ et 1968)}

\begin{tabular}{lcc|cc|cc}
\hline Catégorie professionnelle & \multicolumn{2}{c|}{$\mathbf{1 9 6 4}$} & \multicolumn{2}{c|}{$\mathbf{1 9 6 6}$} & \multicolumn{2}{c}{ En francs } \\
\hline & $\begin{array}{c}\text { Salaire } \\
\text { mensuel }\end{array}$ & $\begin{array}{c}\text { Salaire } \\
\text { horaire }\end{array}$ & $\begin{array}{c}\text { Salaire } \\
\text { mensuel }\end{array}$ & $\begin{array}{c}\text { Salaire } \\
\text { horaire }\end{array}$ & $\begin{array}{c}\text { Salaire } \\
\text { mensuel }\end{array}$ & $\begin{array}{c}\text { Salaire } \\
\text { horaire }\end{array}$ \\
\hline Ouvriers qualifiés, maîtres-valets & 555,75 & 2,36 & 614,20 & 2,78 & 723,80 & 3,22 \\
Ouvriers non qualifiés, domestiques de ferme & 422,85 & 2,01 & 496,70 & 2,37 & 610,50 & 2,80 \\
Ouvrières non qualifiées, servantes de ferme & 240,36 & 1,52 & 380,30 & 1,86 & 409,20 & 2,21 \\
\hline
\end{tabular}

* Les maîtres-valets sont des ouvriers agricoles chargés de l'animation d'une équipe de journaliers ou de domestiques ; ils sont généralement payés au mois.

Sources : d'après DeloRme (1966, p. 20) et Pujol (1969, pp. 105-106).

La revalorisation salariale aussi soudaine que légitime des ouvrières agricoles par le biais de l'application du salaire minimum se traduit par un nouvel effet d'éviction car les gains de productivité générés par la mécanisation réduisent les besoins en maind'œuvre. Si l'emploi salarié en général se restreint, les femmes sont les premières à être écartées du système productif en raison de leur absence de formation technique. Jusqu' aux années 1970, les sessions de formation proposées aux jeunes femmes par les chambres d'agriculture, la Mutualité sociale agricole ou les centres d'information et de vulgarisation agricoles et ménager-agricoles (Civam) sont consacrées aux fonctions traditionnellement dévolues au genre féminin : organisation du ménage, gestion d'un petit élevage, accueil, tenue de comptes, etc. Alors que le salariat féminin progresse en France de $38 \%$ entre 1954 et 1968, le nombre de salariées agricoles régresse des deux tiers et chute de 178000 à 61 000. Dans le même temps, la proportion des femmes dans l'ensemble des salarié.e.s agricoles se contracte de $15,0 \%$ à 10,4 \% . Ce phénomène de baisse se poursuit jusqu' aux années 1980 (INSEE, 1981). L'alignement du Smag sur le Smig, transformé en salaire minimum interprofessionnel de croissance (Smic) suite aux accords dits de «Varenne », est mal vécu par les employeurs agricoles. Même les services de l'Insee se font l'écho de ces récriminations : «Les petites exploitations ne seront peut-être pas en mesure de conserver cette main-d'œuvre permanente sauf si cette dernière accepte d'être rémunérée à des taux inférieurs aux taux réglementaires » (PuJoL, 1969, p. 80). N'est-ce pas là entrouvrir une porte vers des pratiques illégales, à la seule condition qu'elles soient « librement » consenties par les salarié.e.s en question?

La protection sociale assise sur le salaire s'étend au rythme de la salarisation de la société française ; cependant, pour les salariées agricoles, les disparités demeurent. Alors que les contributions aux caisses mutuelles d'assurances sociales agricoles sont indexées sur le salaire pour les salarié.e.s des coopératives et syndicats agricoles, elles dépendent de l'âge et du sexe pour les salarié.e.s des exploitations agricoles (tableau 3). 
Pour les trois catégories d'âge retenues, l'écart de taux de cotisation entre hommes et femmes est de l'ordre de $20 \%$, entérinant de fait la nouvelle norme d'un salaire féminin à $80 \%$ du salaire de base des ouvriers agricoles hommes.

TABLEAU 3 - Cotisations sociales agricoles pour les salarié.e.s des exploitations agricoles en 1956

En francs par jour de travail

\begin{tabular}{lcccccc}
\hline & \multicolumn{2}{c}{ Part ouvrière } & \multicolumn{2}{c}{ Part patronale } & \multicolumn{2}{c}{ Total des cotisations sociales } \\
\hline & Hommes & Femmes & Hommes & Femmes & Hommes & Femmes \\
\hline Moins de 18 ans & 36 & 31 & 52 & 45 & 88 & 76 \\
De 18 à 65 ans & 51 & 44 & 74 & 64 & 125 & 108 \\
Plus de 65 ans & 18 & 16 & 74 & 64 & 92 & 80 \\
\hline
\end{tabular}

Source : Le Paysan du Midi, ${ }^{\circ}$ 535, 20 septembre 1956, p. 2.

Un système de cotisation proportionnelle au salaire, quels que soient l'âge et le sexe, aurait sensiblement modifié le montant de la contribution sociale acquittée par et pour les salariées agricoles. Sur le plan symbolique, le maintien d'une référence au sexe accrédite l'idée que les ouvrières agricoles demeurent des salariées de second rang.

\section{L'allocation de salaire unique, une incitation au retrait du marché du travail}

Le législateur institue le 22 août 1946 une allocation de salaire unique (ASU) attribuée aux ménages ou aux personnes ne bénéficiant que d'un seul revenu professionnel provenant d'une activité salariée. Si cette mesure s'inscrit dans l'esprit de la loi 29 mars 1941, la suppression de toute clause de nationalité autorise les nombreux ménages de travailleurs immigrés à y prétendre. L'octroi de l'ASU a pour préalable l'exclusion officielle sinon effective des femmes de la sphère professionnelle et confère une légitimité sociétale à cette exclusion. Le dispositif rencontre un succès sans précédent auprès des ménages de salariés agricoles : en 1963, 97 \% d'entre eux perçoivent l'ASU (PERROT, 1965, p. 95).

Pour les salarié.e.s en charge de famille, la perte de pouvoir d'achat accumulée en 1949 par rapport à l'avant-guerre est partiellement compensée par la progression des prestations familiales. Beaucoup plus prononcé pour les ménages de deux enfants que pour ceux de cinq, ce rattrapage provient pour l'essentiel de l'ASU. Les prestations familiales agricoles restent cependant inférieures à celles accordées par le régime général de Sécurité sociale car le budget annexe des prestations sociales agricoles (Bapsa) est soumis à la règle de l'équilibre budgétaire. Or le nombre de cotisants diminue alors que, sous l'effet du baby-boom, le nombre de bénéficiaires continue d'augmenter. Le déficit du Bapsa est aggravé par la faiblesse des cotisations. En 1957, les cotisations «allocations familiales » versées par les employeurs plafonnent à 13 milliards de francs auxquels s'ajoutent 7 milliards de taxes additionnelles à l'impôt foncier. Dans le même temps, les prestations familiales s'élèvent à 136 milliards de francs, dont 77 milliards pour les salariés et 59 milliards pour les non-salariés 
(MAUREL, 1959, p. 1009). Les recettes contributives ne couvrent donc que 14,7 \% des dépenses du Bapsa. Les très nombreux ménages de salariés exploitant simultanément un petit bien sont directement affectés par les mesures d'économie mises en œuvre. Les salarié.e.s agricoles exerçant une activité accessoire d'exploitant perçoivent l'ASU seulement si leur propriété a une superficie inférieure au tiers de celle de l'exploitation type s'ils ont deux enfants, à la moitié de cette dernière s'ils en ont au moins trois ${ }^{25}$. Paradoxalement, tout en réclamant le maintien de l'emploi féminin et sa revalorisation salariale, la FNTA/CGT appelle à une extension de l'ASU à tout ménage dont le revenu ne dépasse pas 300000 francs par an.

En 1954, le gouvernement Mendès France maintient l'indexation sur l'indice des prix à la consommation pour les seules allocations familiales ; l'ASU ne suivra plus le coût de la vie. Malgré cela, en raison de la forte natalité, les prestations familiales accordées par ménage allocataire progressent nettement plus vite que l'indice des prix : sur une base 100 en 1949, elles atteignent en 1963 l'indice 337, contre 214 pour les prix de détail (Perrot, 1965, p. 95 et 135). Jusqu'en 1968, un couple ayant deux enfants à charge peut prétendre à une ASU supérieure aux allocations familiales proprement dites. Cette prestation sociale explique en grande partie le retrait (au moins officiel) des jeunes épouses du marché du travail, incitées à se consacrer aux charges domestiques et aux fonctions parentales. Les prestations familiales furent encore plus déterminantes dans le retrait de l'emploi pour les salariées agricoles que pour leurs homologues de l'industrie. Avec respectivement 17,5\% et 20,5\% de femmes officiellement actives, les épouses de salariés agricoles demeurent, en 1968 comme en 1962, celles dont le taux d'activité est le plus faible, très en deçà de la moyenne nationale $(31,6 \%$ et $33,7 \%)$ (BANDERIER, CharRaud, 1975, p. 39).

À partir du $1^{\text {er }}$ juillet 1972, l'ASU n'est plus attribuée que sous conditions de ressources. Sont potentiellement bénéficiaires les seuls ménages dont les revenus n'excèdent pas un plafond de 23040 francs majoré de $25 \%$ par enfant à charge. De plus, les ménages ayant des revenus inférieurs à 2130 fois le taux horaire du Smic majoré de $25 \%$ par enfant à charge bénéficient d'une majoration d'allocation dont le montant, indexé sur le coût de la vie, double entre 1972 et 1976 (ChARRAUd, CHASTAND, 1978, pp. 209-210). Entre 1970 et 1976, les prestations servies à une famille dont le mari perçoit le salaire moyen ouvrier enregistrent un gain de $52 \%$ en pouvoir d'achat pour un seul enfant à charge, de $30 \%$ pour deux enfants à charge et de $23 \%$ pour trois enfants à charge (CHARRAUD, CHASTAND, 1978, pp. 67-69). Le salaire des ouvriers agricoles étant nettement moindre que le salaire ouvrier moyen, le poids des prestations familiales dans les recettes annuelles de leur ménage se trouve majoré d'autant.

La substitution du complément familial à l'ASU au $1^{\text {er }}$ janvier 1978 change toutefois la donne puisque cette nouvelle prestation n'est plus modulée selon le nombre d'enfants et puisque son calcul pénalise moins l'épouse active que la législation antérieure (MARTIN, 1998, pp. 1145-1148). La forte inflation couplée à une revalorisation

25. Circulaire du ministre de l'Agriculture en date du 22 décembre 1949. 
seulement partielle des prestations familiales achève la mutation des comportements féminins. Désormais, les épouses de salariés agricoles comme leurs homologues du secteur industriel et tertiaire se positionnent massivement sur le marché du travail, mais la plupart d'entre elles recherchent un emploi hors de l'agriculture (CANCEILL et al., 1981, pp. 91-94).

\section{Une absence totale de reconnaissance de qualification}

Alors qu'ils avaient été « oubliés » par la loi du 26 juin 1936, la loi du 11 février 1950 accorde enfin aux représentants salariés et employeurs agricoles la possibilité de conclure des accords de salaires et des conventions collectives généralisables à l'ensemble de la profession. La première convention collective concernant les salarié.e.s agricoles est signée dans le Var le 14 juin 1951. Entre 1950 et 1959, 38 départements seulement signent des accords annuels de salaire ou des conventions collectives. De nombreux accords ne donnent par ailleurs jamais lieu à des conventions collectives et certains d'entre eux ne sont pas renouvelés (LANGLOIS, 1962, p. 93). L'adoption de conventions collectives agricoles se généralise durant les années 1960. À la fin de cette décennie, seules demeurent quelques « zones blanches », essentiellement dans le Nord et l'Est. La loi «Auroux » du 13 novembre 1982 contribuera à parachever le paysage des conventions collectives agricoles.

L'application d'une grille de salaire indépendante du genre n'est pas la règle générale. La convention collective agricole actée par les partenaires sociaux dans le Var dissocie par exemple le sort des salariés de celui des salariées. Dans un premier temps, le texte rappelle le principe général égalitaire : «À travail et rendement égaux, les ouvrières reçoivent le même salaire que les ouvriers de la même catégorie. » Mais, dans un second temps, il énonce le paiement des salariées au minimum légal : «Lorsque le travail effectué par une ouvrière est un travail spécifiquement féminin, la rémunération sera déterminée conformément aux dispositions relatives à la fixation du salaire minimum garanti en agriculture ${ }^{26}$. » Lorsque prévaut le paiement à la tâche, la différenciation des rémunérations selon le genre est légitimée par une différence de rendement entre travail masculin et travail féminin, comme dans la convention collective agricole de la Côte-d' $\mathrm{Or}^{27}$.

La différenciation des qualifications selon le genre ne s'amoindrit pas avec les années mais, désormais, les partenaires sociaux manient l'euphémisme. Dans les départements du Midi méditerranéen, alors que le coefficient 100 de base correspondait jusqu'alors à la catégorie « Femmes », à partir de 1962, relève de ce coefficient 100, le «personnel occupé exclusivement aux travaux élémentaires de la viticulture, tels que ramassage des sarments, ébourgeonnage, attachage et couverture des greffes ». Or tous les travaux viticoles ici cités sont dévolus aux femmes depuis toujours. L'appareil

26. Article 18 de la convention collective agricole du Var en date du 14 juin 1951 : « Salaires féminins », Journal officiel, 28 octobre 1953, p. 9666.

27. Journal officiel, 28 octobre 1953, p. 9676. 
statistique lui-même entérine cette absence de qualification des salariées agricoles. Ainsi, dans le cadre des enquêtes annuelles sur la main-d'œuvre agricole publiées par l'Insee tout au long des décennies 1950 et 1960 sur la base d'informations collectées par les inspecteurs départementaux des lois sociales en Agriculture, les salarié.e.s agricoles sont subdivisé.e.s en trois sous-catégories : le «personnel de direction », les « ouvriers qualifiés » et les « ouvrières non qualifiées, servantes de fermes »; la catégorie « ouvrières qualifiées » n'existe pas (VlassenKo, 1956 ; MARCHE, 1958 ; PujoL, 1967).

\section{Le revenu professionnel modique des salariées agricoles intermittentes}

Afin d'illustrer l'intermittence de l'emploi féminin, nous avons dépouillé les livres de comptes et feuilles de paie de deux grands domaines viticoles situés en Camargue (Daladel et Petite Sylve). Dans un premier temps, nous avons relevé les jours de travail et les salaires d'un couple, Angèle et Joseph F., présent sur le domaine de Daladel durant les vingt-quatre années (1949 à 1973) que couvrent les comptes journaliers détaillés disponibles. Uniquement employé pour les vendanges durant l'année 1949, Joseph devient dès 1950 l'un des douze ouvriers agricoles attitrés du domaine. En revanche, jusqu'en 1957, son épouse n'est sollicitée que pour les vendanges. Le couple F. n'ayant qu'un seul enfant, un jeune garçon également prénommé Joseph, Angèle serait vraisemblablement disposée à travailler davantage si le régisseur le lui proposait. En 1958, elle accède au statut d'ouvrière « permanente » même si, comme les autres journalières, elle reste, de fait, employée par intermittence. À partir de 1960, Joseph F. fils est embauché au printemps durant quelques journées, puis surtout pour les vendanges qu'il effectue sur le domaine jusqu'en 1969. Dès les vendanges de 1962, il gagne nettement plus que sa mère et presque autant que son père ${ }^{28}$. Au total, durant les années 1958-1966, période où elle est le plus souvent employée, Angèle ne contribue au revenu familial que dans une proportion variant de $17,5 \%$ (en 1960) à 23,4 \% (en 1961) : la faiblesse des revenus annuels de la journalière agricole se trouve confirmée.

Dans un second temps, nous avons comparé l'employabilité des ouvrières « permanentes » du domaine de Daladel avec celle des ouvrières de Petite Sylve. L'emploi de ces salariées est soumis à de fortes fluctuations, entre ouvrières d'une part, et d'une année sur l'autre d'autre part ; qui plus est, ces fluctuations s'accentuent avec le temps. Durant ces vingt-quatre années, Angèle F. est toujours sollicitée pour les vendanges mais n'est jamais employée durant les mois de juillet, août, octobre et novembre. En hiver, elle ramasse les sarments mais ses quinzaines sont rarement complètes. Au printemps, durant la même quinzaine du mois de mai, elle est employée dix jours une année et seulement deux jours l'année suivante. La situation des autres ouvrières permanentes de ces domaines est assez semblable et, au fil du temps, l'emploi de chacune d'entre elles est toujours plus fluctuant. À l'opposé, les ouvriers agricoles permanents

28. Archives départementales de l'Hérault, 94 J 75, dossier « Bulletins de salaires, Ancienne $C^{\text {ie }}$ du canal de Beaucaire, domaine de Daladel ». 
de ces mêmes exploitations travaillent tout au long de l'année et les écarts de rémunération entre eux n'excèdent jamais $10 \%$. La mécanisation transforme profondément l'employabilité des salariées dans les grandes exploitations. Sur le tableau 4, nous avons rapproché le revenu annuel de l'ouvrière la plus employée sur ces exploitations agricoles camarguaises du salaire moyen d'une ouvrière dans l'industrie. L'écart considérable s'explique par la conjonction de deux variables : une employabilité moindre de l'ouvrière agricole, qui subit plusieurs périodes de «chômage technique », et une rémunération horaire plus faible en raison de l'absence totale de reconnaissance de qualification et d'un salaire minimum moindre pour les salarié.e.s de l'agriculture.

\section{TABLEAU 4 - Rémunération annuelle d'une journalière agricole en Camargue et, en moyenne, d'une ouvrière industrielle}

En francs jusqu'en 1959, en nouveaux francs à partir de 1960

\begin{tabular}{cccccc}
\hline Années & $\begin{array}{c}\text { Rémunération } \\
\text { de la journalière } \\
\text { agricole (1) }\end{array}$ & $\begin{array}{c}\text { Prestations sociales annuelles } \\
\text { touchées par des ménages } \\
\text { comptant deux enfants } \\
\text { à charge (2) }\end{array}$ & $\begin{array}{c}\text { Rapport } \\
(\mathbf{1}) /(\mathbf{2})\end{array}$ & $\begin{array}{c}\text { Rémunération } \\
\text { de l'ouvrière } \\
\text { industrielle (3) }\end{array}$ & $\begin{array}{c}\text { Rapport } \\
(\mathbf{1}) /(3)\end{array}$ \\
\hline 1950 & 120105 & 103680 & 1,16 & 174000 & 0,69 \\
1954 & 132651 & 124200 & 1,07 & 248400 & 0,54 \\
1962 & 2967 & 1584 & 1,87 & 4950 & 0,60 \\
1968 & 2249 & 1872 & 1,20 & 7200 & 0,31 \\
\hline
\end{tabular}

Sources : Archives départementales de l'Hérault, 94 J 1034 (journalière agricole), et MARTIN J. (1998), p. 1135 (ouvrière industrielle).

Il en ressort qu'une ouvrière agricole ayant deux enfants à charge n'a aucun intérêt financier à occuper un emploi pérenne car ce revenu salarial ferait perdre à son ménage le bénéfice de l'ASU. En revanche, cette femme a un intérêt objectif à accepter un emploi temporaire (souvent non déclaré) qui ne la prive d'aucune prestation sociale. En refusant d'être employées plus de deux à trois mois par an, de crainte de perdre le bénéfice de ces prestations sociales, ces ouvrières agricoles s'inscrivent dans la flexibilité que les employeurs agricoles se sont constamment attachés à préserver. Par voie de conséquence, les ouvrières agricoles les plus employées sont célibataires ou jeunes mariées sans enfant. Dès lors, l'emploi agricole féminin se caractérise par un fort turn-over.

Il serait hasardeux de généraliser les points forts émergeant de cette approche monographique tant les paramètres varient selon les productions et les régions. Pour autant, en tous lieux, les femmes apparaissent plus que jamais comme une maind'œuvre potentiellement disponible, toujours mobilisée pour les temps forts des récoltes et sous-utilisée en saison morte. 
Les politiques d'assistance, dont les femmes faisant partie d'un ménage de salariés agricoles ont durablement "bénéficié », n'ont pas débouché sur une amélioration relative de leurs conditions d'existence. Au cours des années 1960-1970, ces ménages sont, plus que jamais, au bas de l'échelle économique et sociale : faiblesse des revenus, bas niveau de formation initiale, accès réduit des enfants aux études supérieures, conditions de logement dégradées, etc. (CANCEILl et al., 1981). « L'institutionnalisation du "précariat" féminin subventionné », pour reprendre les mots de Jean GADREY (2009), trouve ses racines loin dans le temps et les salariées agricoles en furent les premières actrices. Les dispositifs d'assistance mis en œuvre à partir de la dernière décennie du XIX ${ }^{e}$ siècle ont partiellement compensé le déficit de rémunération des ouvrières agricoles. Ces allocations non contributives ${ }^{29}$ ont conféré une légitimité durable à la théorie du salaire féminin comme salaire d'appoint. Si ce phénomène concerne toutes les professions, il revêt une ampleur particulière dans le cas des salariées agricoles en raison de l'intermittence de leur emploi et de la faiblesse de leur rémunération.

L'approche historique du salariat agricole apporte un démenti à l'affirmation pourtant mille fois répétée selon laquelle le travail à temps partiel était exceptionnel en France jusqu'au début des années 1980 (KeRGOAT, 1998, p. 321 ; UlRich, 2009, p. 209). L'emploi intermittent fut séculairement de règle pour les journalières agricoles. Ainsi, l'institutionnalisation du temps partiel par l'ordonnance du 26 mars 1982 ne traduit pas l'émergence d'un phénomène nouveau mais son encadrement administratif dans le contexte de la mensualisation des emplois et son développement dans les secteurs non agricoles. Le temps partiel féminin désormais largement répandu dans de larges sphères du secteur tertiaire (LETABLIER, NICOLE-DrANCOURT, 2007) fut pratique courante pour une fraction du prolétariat agricole pour lequel coexistait déjà le couple emploi intermittent/minima sociaux. Enfin, les épouses de salariés agricoles comme les épouses d'exploitants pâtirent durablement du familialisme particulièrement prégnant dans le milieu agricole (LAGRAVE, 1987, pp. 89-110).

Au terme de ce survol historique, en dépit des garde-fous institués par les lois d'égalité professionnelle hommes/femmes, on soulignera, au regard du recensement général agricole de 2010, combien le salariat agricole féminin reste marqué du sceau de l'intermittence et du travail faiblement qualifié.

29. Sont non contributives toutes les prestations sociales accordées sans contrepartie d'une cotisation d'assurance sociale obligatoire ou non. 


\section{BIBLIOGRAPHIE}

AnOnyme (1933), « Mouvement des salaires en France en 1932 », Bulletin de la Statistique générale de la France, t. XXII, fascicule II, janvier-mars, pp. 229-241.

Auslander L., ZANCARINI-Fournel M. (1995), Différence des sexes et protection sociale (XIX ${ }^{e}$ siècle-XX $X^{e}$ siècle), Saint-Denis, Presses universitaires de Vincennes.

Banderier G., Charraud A. (1975), « Données statistiques sur les familles », Les Collections de l'Insee, Série M, Ménages, $\mathrm{n}^{\circ} 48$.

BARTHEZ A. (1982), Famille, travail et agriculture, Paris, Économica.

Battagliola F. (1999), « Des femmes aux marges de l'activité, au cœur de la flexibilité », Travail, genre et sociétés, $\mathrm{n}^{\circ} 1$, pp. 157-177.

Battagliola F. (2000), « Des aides aux familles aux politiques familiales (1870-1914) », Genèses, nº 40, pp. 144-161.

BECOUARN M.-C. (1975), Le Travail des femmes d'exploitants dans l'agriculture et l'évolution des techniques. Étude de la répartition et des caractéristiques des tâches dans les exploitations agricoles spécialisées, thèse de $3^{\mathrm{e}}$ cycle de sociologie, Université de Tours.

Berlan M., PAInvin R.-M. (1979), Les Agricultrices en France. Étude statistique, École nationale supérieure agronomique de Rennes, Institut national de la recherche agronomique, Économie et sociologie rurales (Station de Paris).

BESSIÈRE C. (2006), Maintenir une entreprise familiale. Enquête sur les exploitations viticoles de la région délimitée Cognac, thèse de doctorat en sociologie, Université Paris V-René Descartes.

Bieganski E., Bousquet S., Danrey J., Pharo P., Simula P., Schaff J.-P., Centre D’études et DE RECHERCHES SUR LES QUALIFICATIONS (1981), Les Salariés agricoles, Paris, La Documentation française.

Bourdelais P. (1981), « Le poids démographique des femmes seules en France (deuxième moitié du XIX ${ }^{\mathrm{e}}$ siècle) », Annales de démographie historique, $\mathrm{n}^{\circ}$ 1, pp. 215-227.

Canceill G., Chastand A., Choquet O. (1981), «Données statistiques sur les familles (Revenus, activité féminine, conditions de vie) », Les Collections de l'Insee, Série M, Ménages, $\mathrm{n}^{\circ} 86$.

Chaineaud C. (2012), La Protection sociale contemporaine de la veuve (1870-1945), Pessac, Presses universitaires de Bordeaux.

CHANDON DE BRIAILlEs R. (1897), «Le vigneron champenois. Les femmes », Revue de viticulture, t. VII, n ${ }^{\circ} 168$, pp. 249-258.

Charraud A., Chastand A. (1978), « Les ressources des familles de salariés de 1970 à 1976 », Les Collections de l'Insee, Série M, Ménages, nº 67.

Cova A. (1997), Maternité et droits des femmes en France (XIX'-XX ${ }^{e}$ siècles), Paris, Anthropos. 
DARPEIX A. (2010), La Demande de travail salarié permanent et saisonnier dans l'agriculture familiale : mutations, déterminants et implications. Le cas du secteur des fruits et légumes français, thèse de doctorat en sciences économiques, Montpellier, SupAgro.

DAVID R. (1897), « La maison Moët-et-Chandon », La Réforme sociale, 4 e série, t. IV, 1 er juillet, pp. 95-105.

Delorme P. (1966), « L'évolution du salariat en agriculture », Économie rurale, n 67, pp. 3-24.

Downs L. L. (2001), L'Inégalité à la chaîne. La division sexuée du travail dans l'industrie métallurgique en France et en Angleterre, 1914-1939, traduit de l'anglais par Éli Commins, Paris, Albin Michel.

Dreyfus M. (2001), Liberté, égalité, mutualité. Mutualisme et syndicalisme, 1852-1967, Paris, les Éditions ouvrières/les Éditions de l'Atelier, Mutualité française.

ESCUDIER J.-L. (1999), « La dynamique de la protection sociale en France de 1850 à 1940 », Sociétés et représentations, $\mathrm{n}^{\circ}$ hors-série, pp. 273-290.

ESCUDIER J.-L. (2014), Contribution à l'histoire des rapports économiques de genre. Application à la viticulture française, 1850-2010, thèse de doctorat en histoire et histoire de l'art, Université de Perpignan Via Domitia.

ESCUDIER J.-L. (2015), « Les houillères au filtre des rapports de genre : des emplois féminins à l'épouse de mineur 1860-1960 », in Aprile S., Oliveira (de) M., Touchelay B., Hoin K.-M. (dir.), Les Houillères entre l'État, le marché et la société. Les territoires de la résilience XVIII XXI ${ }^{e}$ siècles, Villeneuve-d'Ascq, Presses universitaires du Septentrion, pp. 139-153.

ESCUDIER J.-L. (2016), Les Femmes et la Vigne. Une histoire économique et sociale (18502010), Toulouse, Presses universitaires du Midi.

FARCY J.-C., HUBSCHER R. (dir.) (1996), La Moisson des autres : les salariés agricoles aux XIX et $X X^{e}$ siècles, Paris, Créaphis.

FAURE O. (1984), « La médecine gratuite au XIX è siècle : de la charité à l'assistance », Histoire, économie et société, vol. 3, $\mathrm{n}^{\circ} 4$, pp. 593-608.

GADREY J. (2009), «L'institutionnalisation du "précariat" féminin subventionné », Travail, genre et sociétés, ${ }^{\circ} 22$, pp. 159-164.

GoldmAn S. (1982), « Les premières caisses d'allocations familiales en agriculture (19201936) », Colloque sur l'histoire de la Sécurité sociale, Actes du $106^{\mathrm{e}}$ Congrès des sociétés savantes, Perpignan, pp. 83-96.

Guilbert M. (1966), Les Fonctions des femmes dans l'industrie, Paris, Mouton et Cie.

InsEe (1981), Données sociales. Édition 1981, $4^{\text {e }}$ édition, Paris, Insee.

KeRgOAT D. (1998), « La division du travail entre les sexes », in Kergoat J., Boutet J., Jacot H., Linhart D. (dir.), Le Monde du travail, Paris, La Découverte, pp. 319-327.

Lagrave M. (dir.), Leclerc P. (1996), La Sécurité sociale. Son histoire à travers les textes, tome II, 1870-1945, Paris, Association pour l'étude de l'histoire de la Sécurité sociale. 
LAGRAVE R.-M. (1987), « L'agricultrice inclassable : les fonctions sociales du flou statistique », in Lagrave R.-M. (dir.), Celles de la terre. Agricultrice : l'invention politique d'un métier, Paris, Éditions de l'École des hautes études en sciences sociales, pp. 89-110.

LANGLois F. (1962), Les Salariés agricoles en France, Paris, Armand Colin.

LEMIÈRE S. (2006), «Un salaire égal pour un emploi de valeur comparable », Travail, genre et sociétés, $\mathrm{n}^{\mathrm{o}} 15$, pp. 83-100.

Letablier M.-T., Nicole-Drancourt C. (2007), « Le salariat féminin et le modèle de l'intermittence », in Vatin F. (dir.), Le Salariat. Histoire, théorie et formes, Paris, La Dispute, pp. 243-260.

LÉVÊQUE P. (1996), « Les salariés agricoles en Bourgogne au milieu du XIX e siècle », in FARCY J.-C., HuBsCHER R. (dir.), La Moisson des autres : les salariés agricoles aux XIX ${ }^{e}$ et XX ${ }^{e}$ siècles, Paris, Créaphis, pp. 80-99.

MARCHE M. (1958), « Salaires et main-d'œuvre dans l'agriculture en mars 1957 » Études statistiques. Supplément trimestriel du Bulletin mensuel de statistique, $\mathrm{n}^{\mathrm{o}} 2$, pp. 37-43.

Martin G., MARTENOT P. (1909), Contribution à l'histoire des classes rurales en France au XIX ${ }^{e}$ siècle. La Côte-d'Or. Étude d'économie rurale, Dijon, Damidot frères.

Martin J. (1998), « Politique familiale et travail des femmes mariées en France. Perspective historique : 1942-1982», Population, vol. 53, nº 6, pp. 1119-1154.

Martin-Fugier A. (2004), La Place des bonnes. La domesticité féminine à Paris en 1900, Paris, Perrin.

Maruani M., Meron M. (2012), Un siècle de travail des femmes en France 1901-2011, Paris, La Découverte.

MAUREL F. (1959), « Le revenu de l'agriculture en France en 1958-1959 », Études et conjoncture, $\mathrm{n}^{\mathrm{o}} 11, \mathrm{pp} .995-1013$.

Meurs D., Ponthieux S. (2000), « Une mesure de la discrimination dans l'écart de salaire entre hommes et femmes », Économie et statistique, n ${ }^{\circ} 337-338$, pp. 135-158.

Muller L. (2012), « Les écarts de salaire entre les hommes et les femmes en 2009 : le salaire horaire des femmes est inférieur de $14 \%$ à celui des hommes », Dares analyses, $\mathrm{n}^{\circ} 016$.

Ministère de L'Agriculture, Direction de l'Agriculture (1937), Statistique agricole de la France. Résultats généraux de l'enquête de 1929, Paris, Imprimerie nationale.

Ministère de L'Agriculture, OfFice DE RENSEIGNEMENTS AGRiCOLES (1912), Enquête sur les salaires agricoles, Paris, Imprimerie nationale.

OMNÈs C. (1997), Ouvrières parisiennes : marché du travail et trajectoires professionnelles au $X X^{e}$ siècle, Paris, Éditions de l'École des hautes études en sciences sociales.

PAXTON R. O. (1996), Le Temps des chemises vertes. Révoltes paysannes et fascisme rural (1929-1939), Paris, Le Seuil.

Perrot M. (1965), « Données statistiques sur l'évolution des rémunérations salariales de 1938 à $1963 »$, Études et conjoncture, nº 8, pp. 3-137. 
PIÉGAY P. (2007), Domestiques agricoles et servantes de ferme dans les sociétés paysannes (de 1900 aux années 1960), Paris, L'Harmattan.

PraCa E. (2000), Les Sociétés de secours mutuels et leur union dans les Pyrénées-Orientales, $X I X^{e}-X X^{e}$ siècles, Canet, Trabucaire.

Pujol C. (1967), « Les salaires et la main-d'œuvre salariée dans l'agriculture en avril 1966 », Études et conjoncture, $\mathrm{n}^{\circ} 3$, pp. 119-144.

Pujol C. (1969), « Les salaires et la main-d'œuvre salariée dans l'agriculture en avril 1968 », Études et conjoncture, $\mathrm{n}^{\mathrm{o}} 3$, pp. 61-110.

SiLVERA R. (2014), Un quart en moins. Des femmes se battent pour en finir avec les inégalités de salaire, Paris, La Découverte.

THÉBAud F. (1986), Quand nos grands-mères donnaient la vie. La maternité en France dans l'entre-deux-guerres, Lyon, Presses universitaires de Lyon.

ToucAs-Truyen P. (2004), «La mutualité au risque des femmes (1850-1914) », Bulletin d'histoire de la Sécurité sociale, n ${ }^{\circ} 49, \mathrm{pp} .63-78$.

UlRICH V. (2009), «Logiques d'emploi à temps partiel et trajectoires professionnelles des femmes », in Pailhé A., Solaz A. (dir.), Entre famille et travail. Des arrangements de couple aux pratiques des employeurs, Paris, La Découverte, pp. 209-232.

VlassenKo $M^{\text {elle }}(1956)$, «Salaires et main-d'œuvre en agriculture en 1955 », Études statistiques. Supplément trimestriel du Bulletin mensuel de statistique, n ${ }^{\circ}$ 1, pp. 69-76. 